\title{
High failure rate of transungal drug delivery: need for new strategies
}

\author{
"The rapid advancements in research and outcomes in the area of \\ formulations, devices and biochemical engineering techniques to \\ enhance drug penetration necessitate comprehensive progress in \\ transungual drug delivery arena..."
}

First draft submitted: 13 December 2016; Accepted for publication: 20 December 2016; Published online: 31 March 2017

Keywords: nail lacquer $\bullet$ onychomycosis $\bullet$ transungual drug delivery

Nails are the toughest part of the human body. However, due to unabated exposure to environment stress and day-to-day chores, they are highly prone to infections. Onychomycosis is the fungal infection of the nail that can involve one or more of the nail unit components. Infection generally initiates from the distal portion of the toe or finger nail and later spreads to the proximal portion of the nail matrix. The whole width of the nail may be affected. Subsequently, it spreads proximally toward the posterior nail fold and medially to encompass the whole nail bed. In this process, nail can become grossly thickened, and may get completely destroyed [1].

\section{Causes of onychomycosis}

Dermatophytes account for most (90\%) cases of onychomycosis of the toenails and at least $50 \%$ of fingernail. Both dermatophytes (Trichophyton rubrum or Trichophyton mentagrophytes) and nondermatophytes (Scopulariopsisbrevicaulis, Aspergillus spp, Fusarium $s p p$ and sometimes Candida spp) have been identified as etiologic agents of onychomycosis. In addition, mixed infections have been reported, although their significance is less clear [2].

\section{Challenges in treating onychomycosis}

Successful treatment of onychomycosis is a challenging task due to several factors including slow growth of nails, limited vascular access to the nail bed and barrier properties of nail plate that limit the diffusion of a drug molecule through it. The human nail is thought to be composed of nearly 25 layers of tightly bound keratinized cells and to be approximately 100 -fold thicker than stratum corneum. These factors peremptorily make trans-nail penetration of drugs ineffective. In addition, drug transport through the nail plate is also observed to be influenced by physicochemical properties of the drug molecules (e.g., size, shape, charge and hydrophobicity), formulation characteristics (e.g., nature of vehicle, $\mathrm{pH}$ and drug concentration), presence of penetration enhancer, nail properties (e.g., thickness and hydration) as well as interactions between the permeating molecule and keratin network of the nail plate [3].

\section{Management of onychomycosis}

Current approach for treating dermatophyte onychomycosis includes both oral and topical administration of antifungal agents. The main challenge for the researchers is to develop formulations with high penetrating power through the nail unit allowing delivery of effective drug levels to the site(s) of nail infection.

\section{Oral drug therapy}

Among the available antifungal drugs, oral griseofulvin and ketoconazole are not preferred due to their poor efficacy and safety concerns. While, oral terbinafine $(250 \mathrm{mg}$ daily for 12 weeks) and itraconazole 'pulse'

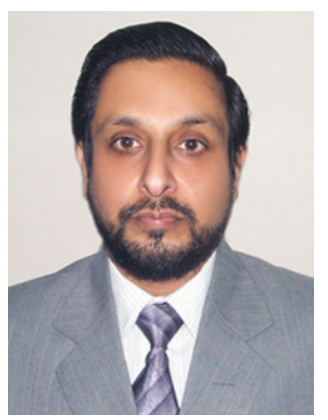

Ashok K Tiwary* Division of Pharmaceutics, Department of Pharmaceutical Sciences \& Drug Research, Punjabi University, Patiala, India

*Author for correspondence: ashokktiwary65@gmail.com

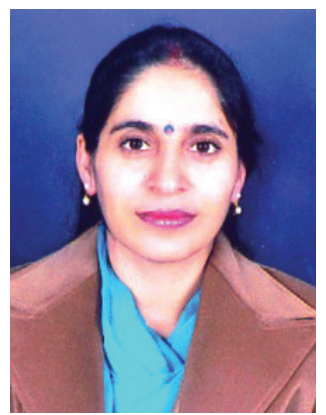

Bharti Sapra

Division of Pharmaceutics, Department of Pharmaceutical Sciences \& Drug Research, Punjabi University, Patiala, India 
therapy (200 mg given twice daily for 1 week per month) have been shown to be the most effective dermatophyte onychomycosis regimens, the latter regimen is not US FDA-approved for toenail onychomycosis. Itraconazole and terbinafine offer mycologic cure rates of only 54 and $70 \%$, respectively [4,5]. Although, fluconazole ( $450 \mathrm{mg}$, once weekly) is also not currently labeled by the FDA for the treatment of onychomycosis, incipient efficacy data are promising. Despite successful in vitro results against the common pathogens causing onychomycosis, in vivo antifungal efficacy remains unsatisfactory due to limited blood supply to the nail bed.

\section{Topical drug therapy}

Topical therapy seems to be an attractive option due to its noninvasiveness and ability to deliver drug(s) to the desired site at a faster rate. Obliteration of systemic adverse events, drug interactions, enhanced patient compliance and reduced cost of treatment are obvious advantages associated with topical therapy. However, active drug from the topical formulation has to permeate and overcome highly restrictive barrier properties of human nail plate. Until 2013, only three topical formulations, Penlac ${ }^{\circledR}$ and $\mathrm{Ciclopoli}^{\circledR}$ (containing ciclopirox) and Loceryl ${ }^{\circledR}$ (containing amorolfine) were approved for the management of onychomycosis in few countries. These products are based on water-insoluble polymers that create a film on the nail surface and require weekly application followed by removal with organic solvents or nail filing. In 2014, two new topical formulations, Kerydin $^{\mathrm{TM}}$ (tavaborole; 5\% solution), Jublia ${ }^{\circledR}$ (efinaconazole; $10 \%$ solution) were approved by the FDA. Application of these solutions without nail debridement is reported to provide mycologic and complete cure rates two- to three-times greater than those reported with ciclopirox $8 \%$ nail lacquer (NL) applied once daily with nail debridement in a very similar patient population [6]. Further, these solutions being alcohol based do not require harsh treatment with organic solvents for removal as required by lacquers.

\section{Formulations}

The process of development of a new chemical entity having the desired pharmacologic/pharmacokinetic profile which allows adequate nail unit penetration is a difficult, lengthy and costly process. Therefore, researchers are more keen in developing a pharmaceutical formulation which would aid in diffusion of drug molecule through the nail plate, thereby achieving effective drug concentration in the nail bed and matrix.

The conventional formulations like solutions, ointments and creams exhibit short residence time.
Moreover, formulations like ointments and creams owing to their hydrophobic nature have found limited application in treatment of nail disorders as it is a wellknown fact that nail plate acts as a hydrogel and any formulation that enhances hydration would definitely increase the permeation of drug through it.

Therefore, formulations that enhance the residence time, increase the hydration level of the nail plate or provide an occlusive effect, are subject of interest for transungual (TU) drug delivery. Formulations like NLs, films and adhesive patches owing to their occlusive properties enhance nail hydration that in turn augments diffusion of permeating molecules by causing distension of the keratin fibers. On the other hand, gels enhance the drug permeation by increasing residence time.

\section{Nail lacquer}

Film forming polymers are a vital ingredient of NLs. The volatile solvents evaporate soon after the application of NLs leaving behind a thin polymeric film on the nail plate. This film acts as a drug depot from which drug penetrates slowly into and through the nail. According to the requirement, the film can be to be removed either mechanically or with the help of solvents. Subsequently, fresh lacquer may be applied to replenish the drug on the site. Penlac ${ }^{\circledR}$ is an FDAapproved NL formulation that contains $8 \%$ ciclopirox for the treatment of onychomycosis [7]. Amorolfine $(5 \%) \mathrm{NL}\left(\right.$ Loceryl $\left.^{\circledR}\right)$ is approved in Australia and UK for the same purpose. Optimization is necessary as permeation of drug(s) from the NL is governed by solubility and diffusion coefficient of the drug in the polymeric film as well as in the nail plate. Few investigators have observed that the penetration of drug increases with increase in its concentration in the formulation. However, other researchers have inferred that the type of formulation affects the effective delivery of drugs $[8,9]$.

It has been observed that NLs containing 2-n-nonyl-1 and 3-dioxolane could deliver approximately sixfold greater econazole to ventral/intermediate nail plate center and approximately 200 -fold greater econazole across the nail plate than formulation without enhancer. This enormous increase in permeation might be due to the development of a resilient film that would have allowed rapid release of permeant for permeation from the lacquer-nail interjunction [10]. Usually, water-insoluble resins have been used in NL formulations because of their better adherence property. However, water-soluble NLs are preferred because of ease of application and removal [11]. The use of hydroxyl propyl chitosan was observed to enhance the rate of permeation of formulated ciclopirox ( 11-fold) and amorolfine ( threefold) 
as compared with that of commercial amorolfine NL. Formulated ciclopirox was found to exhibit better antifungal effect that lasted for approximately $30 \mathrm{~h}$ as compared with commercial as well as formulated amorolfine NL [12]. Hydoxypropyl chitosan based formulation has also been found to inhibit more dermatophytes as compared with commercial ciclopirox NL. In vitro results showed $40-50 \%$ of the applied ciclopirox to penetrate during the first $6 \mathrm{~h}$ irrespective of nails being infected or uninfected, intact or filed [13]. NL containing $\left[{ }^{14} \mathrm{C}\right]$ ketoconazole formulated using dual acrylate-silicone hybrid copolymer was found to be approximately ninefold more effective in enhancing drug permeation than commercial ketoconazole cream. The augmentation of permeation was attributed to film forming and occlusive properties of the vehicle [14]. Recently, the use of hydrophilic (HPMC) and hydrophobic polymer (Eudragit ${ }^{\circledR}$ ) together was found to yield greater contact time and maintained drug level above the MIC for longer duration [15].

\section{Gels}

Gels are highly preferred for the treatment of nail disorders as they are known to hold a considerable amount of water and therefore, can be expected to possess the ability to hydrate the nail plate. The swelling and hydration of the nail plate is said to enhance the drug permeation as a consequence of formation of a less dense structural keratin matrix with large pores. Gels can be considered suitable for iontophoresis for delivering charged drug molecules across the nail plate using electric field. However, the limitations associated with these formulations involve uneasiness during application due to viscosity and their dissipation from the nail plate surface during day-to-day activities [16]. To overcome these drawbacks, thermogelling properties of the formulations have been exploited due to their ability to stay in a liquid state at room temperature but subsequent transition into gel form after application to the nail [17].

\section{Films, patches \& colloid systems}

Unlike conventional dosage forms that shed from the application site and need repeated application, films and patches can be envisaged to maintain the therapeutic drug concentration at the target infected sites for prolonged time periods. The techniques like hotmelt extrusion and film casting hold great promise for this purpose. The bioadhesive patches of different drugs using different polymers can also be used due to their ability to stay fixed at the nail plate. Liposomes being an ensemble of phospholipids and cholesterol behave akin to skin and hence are known to have the potential of enhancing drug penetration through skin.
Researchers are now exploiting this delivery system for TU route. The nanocapsules and nanoemulsions containing Melaleuca alternifolia (tea tree) essential oil against $T$. rubrum showed higher in vitro antifungal efficacy as compared with conventional emulsion formulation [18]. The permeation of a novel antifungal agent, EV-086K (highly lipophilic), through nail by a colloidal carrier system was found to be approximately ninefold higher than the solution form [19].

\section{Laser treatment}

In addition to the above mentioned formulations, a number of laser devices are now approved by the US FDA for the treatment of onychomycosis. The first laser device to be approved was the short-pulse neodymium yttrium-aluminum garnet (Nd:YAG) 1064 device. Thermal damage was believed to be responsible for the fungistatic or fungicidal activities by these devices. However, tolerability to human patients, immunologic effect in the host and lack of evidence of inhibition of fungal activity after direct laser exposure forced the FDA to label such lasers with the indication "temporary increase of clear nail in onychomycosis". Hence, these devices are not considered to be sufficiently effective to warrant their use as first-line monotherapy for these infections.

\section{Photodynamic therapy}

Research by a number of investigators has demonstrated photodynamic therapy (PDT) to offer a promise in the treatment of onychomycosis. The mechanism of action involves fungal absorption of a chemical, which is metabolized into a photosensitizer, followed by exposure to a specific wavelength light that results in subsequent reactive oxygen species and fungal death. PDT is not currently a practical option for widespread use in treating onychomycosis, and to date no PDT system has been approved by FDA for this indication. However, PDT may be considered when onychomycosis is chronic and is refractory to other therapies, especially when the infection involves fewer nails and less common and more resistant organisms, such as nondermatophyte molds. Future discoveries may alter the paradigm dramatically.

\section{Conclusion}

Amongst different formulations used as nail cosmetics, nail hardeners and hypoallergenic NLs seem to provide an interesting platform in future for use in the management of ungual problems. Nail hardeners can be applied as a base coat for the purpose of strengthening the nail plate, as brittleness is a common problem in onychomycosis. They may contain titanium-silicone-zirconium polymers, polytef, nylon, calcium and biotin as the 
components. Hypoallergenic NLs, which are formaldehyde resin free, may prevent paronychia and dermatitis can also be used in future to reduce the prevalence of nail diseases. Cuticle oils can be used by the persons who are exposed to conditions like overwetting so as to prevent or delay the conditions which may lead to nail infections. Even $\mathrm{pH}$ adjusters can be used as one of the ingredients of nail cosmetics so as to avoid favorable $\mathrm{pH}$ conditions that may lead to nail-fungal infections.

The field of TU drug delivery is still in its juvenile age. Ample opportunities exist for researchers to develop new strategies for effective drug delivery in this area. This will require conceptualizing beyond traditional dosage forms and may lead to different approaches and dosage forms for dealing with ungual problems at different stages and due to different causes. The fact that latestage clinical trials of topical antimycotics authenticate the research in this field makes it imperative to design newer methods for testing efficacy of the developed

\section{References}

1 Roberts DT. Onychomycosis: current treatment and future challenges. Br. J. Dermatol. 141, 1-4 (1999).

2 Summerbell RC. Epidemiology and ecology of onychomycosis. Dermatology 194, 32-36 (1997).

3 Sun Y, Liu JC, Wang JCT. Nail penetration: focus on topical delivery of antifungal drugs for onychomycosis treatment. In: Percutaneous Absorption: Drugs, Cosmetics, Mechanisms, Methodology (3rd Edition). Bronaugh RL, Maibach HI (Eds). Marcel Dekker Inc, NY, USA 759 (1999).

4 Sporanox (itraconazole) [package insert]. Janssen, Titusville, NJ, USA (2012).

5 Lamisil (terbinafine $\mathrm{HCl}$ ) [package insert]. Novartis, East Hanover, NJ, USA (2012).

6 Seebacher C, Brasch J, Abeck D et al. Onychomycosis. Mycoses 50, 321-327 (2007).

7 Gupta AK, Malkin KF. Ciclopirox nail lacquer and podiatric practice. J. Am. Podiatr. Med. Assoc. 90, 502-507 (2000).

8 Pittrof F, Gerhards J, Erni W, Klecak G. Loceryl nail lacquer - realization of a new galenical approach to onychomycosis therapy. Clin. Exp. Dermatol. 17, 26-28 (1992).

9 Mertin D, Lippold BC. In-vitro permeability of the human nail and of a keratin membrane from bovine hooves: penetration of chloramphenicol from lipophilic vehicles and a nail lacquer. J. Pharm. Pharmacol. 49, 241-245 (1997).

10 Hui X, Chan TC, Barbadillo S, Lee C, Maibach HI, Wester RC. Enhanced econazole penetration into human nail by 2-n-nonyl-1,3-dioxolane. J. Pharm. Sci. 92, 142-148 (2003).

11 Monti D, Saccomani L, Chetoni P, Burgalassi S, Saettone MF, Mailland F. In vitro transungual permeation of ciclopirox from a hydroxypropyl chitosan-based, watersoluble nail lacquer. Drug Dev. Ind. Pharm. 31, 11-17 (2005). dosage form earlier during the product development stage. The rapid advancements in research and outcomes in the area of formulations, devices and biochemical engineering techniques to enhance drug penetration necessitate comprehensive progress in TU drug-delivery arena. On the basis of few reports available in prior article, it will not be out of context to believe that the success of TU delivery lies in untangling the challenges of nail penetration enhancement.

\section{Financial \& competing interests disclosure}

The authors have no relevant affiliations or financial involvement with any organization or entity with a financial interest in or financial conflict with the subject matter or material discussed in the manuscript. This includes employment, consultancies, honoraria, stock ownership or options, expert testimony, grants or patents received or pending or royalties.

No writing assistance was utilized in the production of this manuscript.

12 Monti D, Saccomani L, Chetoni P et al. Hydrosoluble medicated nail lacquers: in vitro drug permeation and corresponding antimycotic activity. Br. J. Dermatol. 162, 311-317 (2010).

13 Togni G, Mailland F. Antifungal activity, experimental infections and nail permeation of an innovative ciclopirox nail lacquer based on a water-soluble biopolymer. J. Drugs Dermatol. 9, 525-539 (2010).

14 Hafeez F, Hui X, Chiang A, Hornby S, Maibach H. Transungual delivery of ketoconazole using novel lacquer formulation. Int. J. Pharm. 456, 357-361 (2013).

15 Patel MM, Vora ZM. Formulation development and optimization of transungual drug delivery system of terbinafine hydrochloride for the treatment of onychomycosis. Drug Deliv. Transl. Res. 6, 263-275 (2016).

16 Repka MA, Mididoddi PK, Stodghill SP. Influence of human nail etching for the assessment of topical onychomycosis therapies. Int. J. Pharm. 282, 95-106 (2004).

17 Nogueiras-Nieto L, Begona Delgado-Charro M, OteroEspinar FJ. Thermogelling hydrogels of cyclodextrin/ poloxamer polypseudorotaxanes as aqueous-based nail lacquers: application to the delivery of triamcinolone acetonide and ciclopiroxolamine. Eur. J. Pharm. Biopharm. 83, 370-377 (2013).

18 Flores FC, de Lima JA. Antifungal activity of nanocapsule suspensions containing tea tree oil on the growth of Trichophyton rubrum. Mycopathologia 175, 281-286 (2013).

19 Naumann S, Meyer JP, Kiesow A, Mrestani Y, Wohlrab J, Neubert RH. Controlled nail delivery of a novel lipophilic antifungal agent using various modern drug carrier systems as well as in vitro and ex vivo model systems. J. Control Rel. 180, 60-70 (2014). 